\section{The Pharmacy Leadership Field Guide: Cases and Advice for Everyday Situations}

DeCoske MA, Tryon JE, White SJ. American Society of Health-System Pharmacists, Bethesda, Maryland, 2011. ISBN 978-1-58528-249-4. Softcover, 250 pages. US\$31 for ASHP members (US\$39 for nonmembers).

The stated purpose of this text is "to present pertinent leadership concepts using real-life cases that apply to pharmacy students, residents and new practitioners." The text consists of 9 chapters with titles such as "Becoming a Leader", "Leading Yourself", and "Working Efficiently". Each chapter is further broken down into subtopics that deal with contemporary leadership issues. Each subtopic is addressed through the use of a case in which the protagonist, a new practitioner, finds him- or herself in a challenging situation, with each scenario being set up to allow discussion of certain leadership and management skills. Advice on how to handle the challenges presented in each case is provided first by one of several veteran mentors and then by one of a number of new pharmacy leaders at an earlier stage of their career development. The text also includes additional material at the end of each chapter, such as recommended reading material, self-assessment exercises, and even a few crossword puzzles and word games based on material covered in the chapter.

This book contains a lot of accumulated leadership wisdom. It will undoubtedly be helpful to new practitioners who are interested in pursuing a leadership and/or management career path. The case studies are realistic, and, as is typically the case in the real world, each case usually requires application of a number of different leadership skills. This is both a strength and a weakness of the text. After a few cases, the advice from the mentor and the new leader begins to have a repetitive ring. While such repetition may help to reinforce the principles being presented, readers may find themselves skipping over advice that seems to add little to what has already been said about similar issues in previous cases. Each case also has a section comparing what the protagonist "might be thinking" and what he or she "might be reasoning", a distinction that I found a bit confusing. I expected that the "might be thinking" component would identify the emotional response that a new practitioner was experiencing, whereas the "might be reasoning" part would address a reasoned response after some thought had been given to the issue, but there seemed to be a lack of consistency in terms of what appeared under these 2 headings. After a few cases, I found myself skipping over these sections.

Although there is a lot of good leadership advice in this book, the format in which it is presented makes it a bit of a challenge to stay engaged as a reader.

Kevin W Hall, BScPharm, PharmD

Faculty of Pharmacy and Pharmaceutical Sciences

University of Alberta

Edmonton, Alberta

Dr Hall is also an Associate Editor with the CJHP.

\title{
Analysis of Orders for QTc-Prolonging Medication for Intensive and Cardiac Care Unit Patients with Pre- existing QTc Prolongation (QTIPPP Study): Correction
}

In the byline for an article presenting the results of a study of QTc-prolonging medications administered to patients with pre-existing prolongation of the QTc interval, the order of authors' names was incorrect. ${ }^{1}$ The intended order appears here:

Sayako Yokoyama, Vincent H Mabasa, Damen Man, and John Martyn

The contact information for correspondence related to the article is correct as published.

\section{Reference}

1. Mabasa VH, Yokoyama S, Man D, Martyn J. Analysis of orders for QTc-prolonging medication for intensive and cardiac care unit patients with pre-existing QTc prolongation (QTIPPP Study). Can J Hosp Pharm 2011;64(6):412-418. 